Gravitomagnetism and relative observer clock effects

This article has been downloaded from IOPscience. Please scroll down to see the full text article.

2001 Class. Quantum Grav. 18653

(http://iopscience.iop.org/0264-9381/18/4/306)

View the table of contents for this issue, or go to the journal homepage for more

Download details:

IP Address: 128.206.162.204

The article was downloaded on 23/09/2010 at 22:18

Please note that terms and conditions apply. 


\title{
Gravitomagnetism and relative observer clock effects
}

\author{
Donato Bini ${ }^{1,2}$, Robert T Jantzen ${ }^{2,3}$ and Bahram Mashhoon ${ }^{4}$ \\ ${ }^{1}$ Istituto per Applicazioni della Matematica CNR, I-80131 Napoli, Italy \\ ${ }^{2}$ ICRA International Center for Relativistic Astrophysics, University of Rome, I-00185 Rome, \\ Italy \\ ${ }^{3}$ Department of Mathematical Sciences, Villanova University, Villanova, PA 19085, USA \\ ${ }^{4}$ Department of Physics and Astronomy, University of Missouri-Columbia, Columbia, \\ MO 65211, USA
}

Received 28 October 2000, in final form 7 December 2000

\begin{abstract}
The gravitomagnetic clock effect and the Sagnac effect for circularly rotating orbits in stationary axisymmetric spacetimes are studied from a relative observer point of view, clarifying their relationships and the roles played by special observer families. In particular, Semerák's recent characterization of extremely accelerated observers in terms of the two-clock clock effect is shown to be complemented by a similarly special property of the single-clock clock effect.
\end{abstract}

PACS number: 0420C

\section{Introduction}

Stationary axially symmetric spacetimes, especially the Kerr spacetime as the model for rotating black holes, have proven to be a useful arena for studying various relativistic effects. In particular, the consequences of rotation in relativity compared with the simpler picture of Newtonian physics are naturally explored within this class of spacetimes, other interesting cases for this purpose being the Gödel spacetime in its stationary axially symmetric form and the Minkowski spacetime in uniformly rotating cylindrical coordinates.

Over the past decade a significant effort from various points of view has been invested in developing a careful analysis of test particle and test gyroscope motion in rotating black hole spacetimes in connection with the definition of inertial forces in general relativity as well as in clarifying the meaning of 'non-rotation' in a 'rotating' spacetime. The tools of gravitoelectromagnetism $[1,2]$, i.e. spacetime splitting techniques based on families of test observers, can be very helpful in this investigation. Each such family measures spacetime quantities relative to its own local space and time directions, thus performing a 'relative observer analysis'. Here we study the so-called 'gravitomagnetic clock effect' and the related Sagnac effect using these tools. This is especially interesting for the clock effect since there are actually attempts under consideration to measure it.

One must distinguish three distinct gravitomagnetic clock effects for a pair of oppositely rotating circular geodesic test particles (oppositely rotating with respect to an intermediate observer): 
(a) the observer-dependent single-clock clock effect: the difference between the periods of the oppositely rotating geodesic test particles as measured by the observer's clock [3];

(b) the observer-dependent two-clock clock effect: the difference between the periods of the oppositely rotating geodesic test particles as measured by their own clocks for one revolution with respect to an observer [4-6]; and

(c) the observer-independent two-clock clock effect: the difference between the periods of the oppositely rotating geodesic test particles as measured by their own clocks between two crossing events [7-10].

In the first two cases, for a given observer, one compares the periods of one revolution of these orbits (starting from and returning to the same observer worldline) measured either by the observer's own clock (single-clock effect) or by the clocks carried along the two orbits (two-clock effect). In the third case, no observer enters the calculation.

Here we draw some connections between these effects and the Sagnac and desynchronization effects and the usual symmetry-adapted coordinates in these spacetimes, while extending some previous work. In particular, we extend Semerák's result [6] for the observer-dependent two-clock clock effect for extremely accelerated observers in the Kerr and van Stockum spacetimes to general stationary axially symmetric spacetimes and explain its connection to the single-clock clock effect, where these observers also play a special role. Finally, the observer-independent two-clock clock effect is illustrated for the Gödel spacetime and all the clock effects for the equatorial plane of the Kerr-Taub-NUT spacetime.

\section{Metric splitting}

Consider the spacetime metric

$$
\mathrm{d} s^{2}=\mathrm{d} s_{(t, \phi)}^{2}+g_{r r} \mathrm{~d} r^{2}+g_{\theta \theta} \mathrm{d} \theta^{2},
$$

with the metric of the circular orbit cylinder written in the respective coordinate, threading and slicing notation

$$
\begin{aligned}
\mathrm{d} s_{(t, \phi)}^{2} & =g_{t t} \mathrm{~d} t^{2}+2 g_{t \phi} \mathrm{d} t \mathrm{~d} \phi+g_{\phi \phi} \mathrm{d} \phi^{2} \\
& =-M^{2}\left(\mathrm{~d} t-M_{\phi} \mathrm{d} \phi\right)^{2}+\gamma_{\phi \phi} \mathrm{d} \phi^{2} \\
& =-N^{2} \mathrm{~d} t^{2}+g_{\phi \phi}\left(\mathrm{d} \phi+N^{\phi} \mathrm{d} t\right)^{2},
\end{aligned}
$$

where in the case of Kerr spacetime, $\{t, r, \theta, \phi\}$ are the usual Boyer-Lindquist coordinates adapted to the family of static observers (or threading observers, with 4-velocity $m=M^{-1} \partial_{t}$ and worldlines along the time coordinate lines) and to the ZAMOs (or slicing observers, with 4velocity $n=N^{-1}\left(\partial_{t}-N^{\phi} \partial_{\phi}\right)$ and worldlines orthogonal to the time coordinate hypersurfaces). The spacelike unit vectors giving the (increasing $\phi$ ) angular direction in the local rest space of these observers are

$$
\bar{m}=\gamma_{\phi \phi}^{-1 / 2}\left(\partial_{\phi}+M_{\phi} \partial_{t}\right), \quad \bar{n}=g_{\phi \phi}^{-1 / 2} \partial_{\phi} .
$$

The measurement of spacetime quantities in terms of the $1+3$ orthogonal decomposition associated with the threading observers (moving along the time lines-the 'threading' of spacetime) is called the threading point of view, while the corresponding measurement process associated with the slicing observers (moving orthogonal to the time hypersurfaces-the 'slicing' of spacetime) is called the slicing point of view.

For other spacetimes in this general symmetry class, like the cylindrically symmetric Gödel or van Stockum spacetimes [5], for example, a more convenient notation would use cylindricallike $\rho-z$ coordinates instead of spherical-like $r-\theta$ coordinates and the Lewis-Papapetrou form 
of the metric. In any case, when restricting this metric to the equatorial plane $\theta=\pi / 2$ in Kerr, as will be done for most of our explicit work, a simple correspondence $-\partial_{\theta} \rightarrow \partial_{z}$ and $r \rightarrow \rho$ will map one form onto the other.

All of the Kerr-specific discussion below applies to the entire type-D many-parameter family of solutions treated by Carter [11,12]. For these spacetimes an additional preferred observer 4-velocity $u_{\text {(car) }}$ [13] is the direction of the intersection of the $t-\phi$ plane in the tangent space with the plane spanned by the two independent principal null directions of the Weyl tensor; in this frame the electric and magnetic parts of the Weyl tensor and of the electromagnetic field tensor if present are proportional (the form of the Kerr metric adapted to the Carter observers is just the Boyer-Lindquist difference of squares form of the $t-\phi 2$ metric given in Misner et al [14, equation (33.2)]). Furthermore, in this class of spacetimes the timelike Killing vector $\partial_{t}$ along $m$ is assumed to be the unique such field which has unit norm at spatial infinity in the direction away from the symmetry axis, i.e. corresponding to the distantly non-rotating observers. For all spacetimes under consideration, the spacelike Killing vector field $\partial_{\phi}$ is the unique such field with closed integral curves.

The single-clock clock effect discussion requires that there exist a pair of oppositely rotating geodesics with respect to the distinct preferred observer $m$, which excludes the case that the Killing direction $m$ itself is geodesic, as occurs in the Gödel spacetime. However, the two-clock clock effect can still be considered there.

The lapse and shift notation in the two points of view has the following dual correspondence:

$$
\partial_{t}=N n+N^{\phi} \partial_{\phi}, \quad \mathrm{d} t=M^{-1}\left(-m^{\mathrm{b}}\right)+M_{\phi} \mathrm{d} \phi,
$$

where $m^{b}$ is the 'index-lowered' 1 -form corresponding to the vector field $m$. If $\tau_{u}, u=m, n$, is a proper time parametrization of the threading and slicing worldlines, then the lapse functions relate differential increments of coordinate time to proper time along the worldline

$$
\mathrm{d} \tau_{m}=M \mathrm{~d} t, \quad \mathrm{~d} \tau_{n}=N \mathrm{~d} t .
$$

For constant speed circular orbits, the same relationships apply to finite time intervals.

The shift fields describe the tilting of the local time and space directions of the slicing and threading observers, respectively, from the time coordinate lines and hypersurfaces. In the slicing point of view the local time direction of the slicing observers is the direction for which the 1 -form $\bar{n}^{b} \propto \mathrm{d} \phi+N^{\phi} \mathrm{d} t$ vanishes (equivalent to orthogonality to the angular direction $\bar{n}$ )

$$
\mathrm{d} \phi=-N^{\phi} \mathrm{d} t \quad \rightarrow \quad \mathrm{d} \phi / \mathrm{d} t=-N^{\phi}=\zeta_{(\mathrm{sl})},
$$

which gives the rate of change of angle with respect to time (angular velocity) of the slicing observer worldline following the local time direction.

In the threading point of view, the local spatial angular direction (in the local rest space of the threading observer) is the direction for which the 1-form $m^{b} \propto \mathrm{d} t-M_{\phi} \mathrm{d} \phi$ vanishes (equivalent to orthogonality to the direction $m$ )

$$
\mathrm{d} t=M_{\phi} \mathrm{d} \phi \rightarrow \mathrm{d} t / \mathrm{d} \phi=M_{\phi}=\bar{\zeta}_{(\mathrm{th})}{ }^{-1},
$$

which gives the rate of change with respect to the angle of the time coordinate along a circular curve which is spatial with respect to the threading observers (an inverse angular velocity). The coordinate time difference which accumulates along such a curve after one complete revolution with respect to the threading observers, which may be converted into a proper time with respect to those observers by multiplication by the lapse function, is called the synchronization gap [15]

$$
\Delta t_{(\mathrm{SG})}=2 \pi M_{\phi}, \quad \Delta \tau_{(\mathrm{SG})}=2 \pi M M_{\phi} .
$$


Table 1. The lapse and the non-zero observer-adapted components of the shift and spatial metric in both the threading and slicing points of view are given for the Kerr black hole spacetimes on the equatorial plane, with the abbreviation $\Delta=r^{2}-2 \mathcal{M} r+a^{2}$. The assumption $a>0$ corresponds to the hole rotating in the counterclockwise (forward) angular $\phi$-direction. Angular velocities determining the local time direction and local forward angular direction are also given for these observers and the Carter observers.

\begin{tabular}{ll}
\hline$N=\left(-g^{t t}\right)^{-1 / 2}$ & $\sqrt{r \Delta /\left(r^{3}+a^{2} r+2 a^{2} \mathcal{M}\right)}$ \\
$N^{\phi}$ & $-2 a \mathcal{M} /\left(r^{3}+a^{2} r+2 a^{2} \mathcal{M}\right)$ \\
$g_{\phi \phi}$ & $\left(r^{3}+a^{2} r+2 a^{2} \mathcal{M}\right) / r$ \\
$M=\left(-g_{t t}\right)^{1 / 2}$ & $\sqrt{(r-2 \mathcal{M}) / r}$ \\
$M_{\phi}=-g_{t \phi} / g_{t t}$ & $-2 a \mathcal{M} /(r-2 \mathcal{M})$ \\
$\gamma_{\phi \phi}=g_{\phi \phi}-\left(g_{t \phi}\right)^{2} / g_{t t}$ & $r \Delta /(r-2 \mathcal{M})$ \\
$g_{r r}$ & $r^{2} / \Delta$ \\
$g_{\theta \theta}$ & $r^{2}$ \\
$\zeta_{(\mathrm{sl})}, \bar{\zeta}_{(\mathrm{sl})}$ & $-N^{\phi}, 0$ \\
$\zeta_{(\mathrm{th})}, \bar{\zeta}_{\text {(th) }}$ & $0, M_{\phi}^{-1}$ \\
$\zeta_{(\mathrm{car})}, \bar{\zeta}_{(\mathrm{car})}$ & $a /\left(r^{2}+a^{2}\right), 1 / a$ \\
\hline
\end{tabular}

Circular orbits in the Kerr spacetime equatorial plane will be used to illustrate the various ideas introduced for the entire symmetry class of spacetimes under consideration. The metric coefficients, lapse and shift factors, and angular velocities of the local time and angular directions for this case are given explicitly in table 1.

\section{Circular orbit geometry}

Consider a generic observer with 4-velocity $U$ following a circular orbit worldline, moving along the $\phi$ direction with constant angular velocity $\zeta=\mathrm{d} \phi / \mathrm{d} t=\dot{\phi}$. Its worldlines may be taken as a new threading associated with the new comoving coordinates defined by

$$
\tilde{t}=t, \quad \tilde{\phi}=\phi-\zeta t .
$$

The orthogonal decomposition of the metric adapted to this observer corresponds to the new threading decomposition

$$
\mathrm{d} s_{(t, \phi)}^{2}=-\tilde{M}^{2}\left(\mathrm{~d} \tilde{t}-\tilde{M}_{\tilde{\phi}} \mathrm{d} \tilde{\phi}\right)^{2}+\tilde{\gamma}_{\tilde{\phi} \tilde{\phi}} \mathrm{d} \tilde{\phi}^{2},
$$

where the new threading lapse and shift fields are

$$
\tilde{M}=\Gamma^{-1}, \quad \tilde{M}_{\tilde{\phi}}=\left(g_{\phi \phi} \zeta+g_{t \phi}\right) \Gamma^{2}=(\bar{\zeta}-\zeta)^{-1} .
$$

The new observer 4-velocity $U$ and its orthogonal partner $\bar{U}$ in both sets of coordinates are

$U=\Gamma\left(\partial_{t}+\zeta \partial_{\phi}\right)=\tilde{M}^{-1} \partial_{\tilde{t}}, \quad \bar{U}=\bar{\Gamma}\left(\partial_{t}+\bar{\zeta} \partial_{\phi}\right)=\tilde{\gamma}_{\tilde{\phi} \tilde{\phi}}^{-1 / 2}\left(\partial_{\tilde{\phi}}+\tilde{M}_{\tilde{\phi}} \partial_{\tilde{t}}\right)$

The quantity $\bar{\zeta}^{-1}$ is the rate of change of coordinate time per angle along the spatial angular direction of this new observer (along which $\mathrm{d} t-\bar{\zeta}^{-1} \mathrm{~d} \phi=0$ ), associated with the desynchronization effect. The 'coordinate gamma factor' $\Gamma=\mathrm{d} t / \mathrm{d} \tau_{U}>0$ is defined by

$$
\begin{aligned}
\Gamma^{-2} & =-\left[g_{t t}+2 \zeta g_{t \phi}+\zeta^{2} g_{\phi \phi}\right]=-g_{\phi \phi}\left(\zeta-\zeta_{-}\right)\left(\zeta-\zeta_{+}\right) \\
& =M^{2}\left(1-M_{\phi} \zeta\right)^{2}-\gamma_{\phi \phi} \zeta^{2}=N^{2}-g_{\phi \phi}\left(\zeta+N^{\phi}\right)^{2}
\end{aligned}
$$


The reciprocal $\tilde{M}=\Gamma^{-1}$ is the lapse function relating increments of coordinate time to a proper time parameter $\tau_{U}$ along the new threading worldline

$$
\mathrm{d} \tau_{U}=\Gamma^{-1} \mathrm{~d} t
$$

and for constant speed circular orbits, the same relationship applies to finite time intervals.

The timelike condition for the unit 4-velocity $U$ requires $\Gamma^{-2}>0$, constraining $\zeta$ to belong to the interval $\left(\zeta_{-}, \zeta_{+}\right)$between the roots of the quadratic equation $\Gamma^{-2}=0$ in $\zeta$ corresponding to null directions, namely

$\zeta_{ \pm}=\left[-g_{t \phi} \pm\left(g_{t \phi}^{2}-g_{\phi \phi} g_{t t}\right)^{1 / 2}\right] / g_{\phi \phi}=\left[M_{\phi} \pm M^{-1} \gamma_{\phi \phi}^{1 / 2}\right]^{-1}=-N^{\phi} \pm N g_{\phi \phi}^{-1 / 2}$.

A zero-rest-mass test particle (photon) has instead a 4-momentum vector of the same form

$$
P_{ \pm}=\Gamma_{(\text {null })}\left(\partial_{t}+\zeta_{ \pm} \partial_{\phi}\right)
$$

but with an arbitrary normalization factor $\Gamma_{\text {(null) }}$. Circular orbits for which $\zeta>0$ or $\zeta<0$ will be referred to, respectively, as corotating or counterrotating (with respect to the 'static observers' of the original coordinate grid).

Figure 1 shows the $t-\phi$ plane in the tangent space at a typical radius (where both circular geodesics are timelike) in the equatorial plane in the region of Kerr spacetime superimposed on the $t-\phi$ cylinder, with its tilted vertical axis along $t$, tilted left with respect to the axis along $n$ shown perpendicular to the $\phi$-direction. The null vectors $P_{ \pm}=n \pm \bar{n}$ are shown with the normalization $\Gamma_{\text {(null) }}=N^{-1}$, where $\bar{n}$ is a unit vector along the $\phi$-coordinate line. The other two angular directions $\bar{m}$ and $\bar{u}_{\text {(car) }}$ are also shown at the end of one revolution of a curve which is spatial with respect to the associated observer.

The coordinate time difference $\Delta t$ after one such revolution $\phi: 0 \rightarrow 2 \pi$ along the curve with direction $\bar{m}$ is called the synchronization gap for the static observer $m$, easily converted into a proper time difference measured by this observer.

The physical components of the velocities measured by the threading and slicing observers for motion along circular orbits are related to the coordinate angular velocity by linear or fractional linear transformations

$v(U, m)^{\hat{\phi}}=\gamma_{\phi \phi}{ }^{1 / 2} \zeta /\left[M\left(1-M_{\phi} \zeta\right)\right], \quad v(U, n)^{\hat{\phi}}=g_{\phi \phi}{ }^{1 / 2}\left(\zeta+N^{\phi}\right) / N$

and

$\zeta=M v(U, m)^{\hat{\phi}} /\left[\gamma_{\phi \phi}{ }^{1 / 2}+M M_{\phi} v(U, m)^{\hat{\phi}}\right]=-N^{\phi}+N g_{\phi \phi}{ }^{-1 / 2} v(U, n)^{\hat{\phi}}$.

Here the hatted index notation $\hat{\phi}$ refers to the component along the unit vector $\bar{m}$ or $\bar{n}$ of (2.3) giving the forward (increasing $\phi$ ) angular direction in the local rest space of each point of view. Note that when the shift is non-zero, test particle motions with angular velocities of equal magnitude but opposite sign lead to physical velocities which do not have the same magnitude and vice versa. When $v(U, u)^{\hat{\phi}}= \pm 1$, the latter equation reduces to equation (3.7). The broken line in figure 1 between the tips of $P_{ \pm}$in the tangent space contains the slicing relative velocities associated with the various observer worldlines as the connecting vectors between the tip of $n$ and the points of intersection of those worldlines (actually their 4-velocity vectors) with the broken line.

The 'coordinate' gamma factor is easily expressed in terms of the usual Lorentz gamma factor associated with these relative velocities

$$
\begin{aligned}
\Gamma & =\gamma(U, m) /\left[M\left(1-M_{\phi} \zeta\right)\right] \equiv \Gamma(U, m) \\
& =\gamma(U, n) / N \equiv \Gamma(U, n) .
\end{aligned}
$$




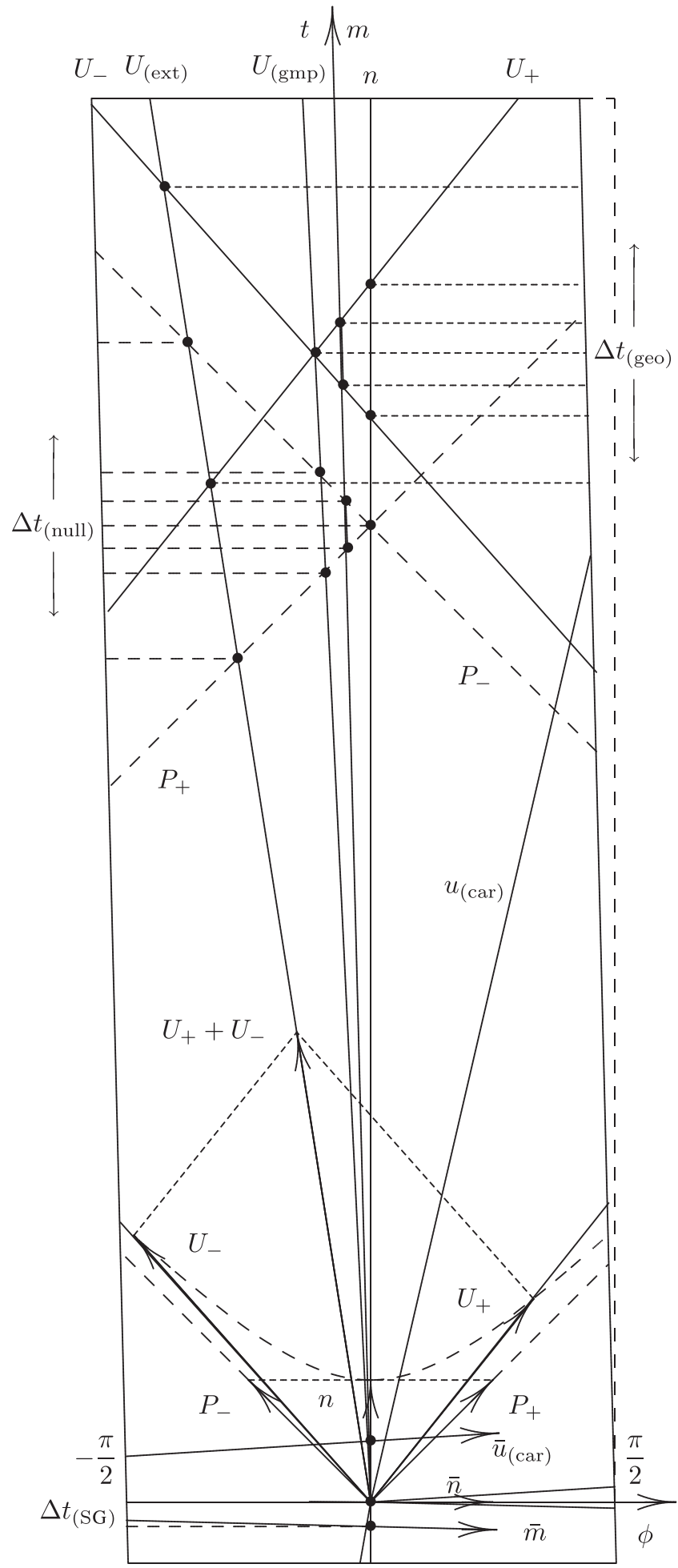

Figure 1. This spacetime diagram shows the Sagnac and clock effects in the equatorial plane of the Kerr spacetime at a typical radius where both circular geodesics (labelled by $U_{+}$and $U_{-}$) are still timelike. The forward and backward circular photon orbits (labelled by $P_{+}$and $P_{-}$) are at a $45^{\circ}$ angle with respect to the horizontal direction. The front half $(-\pi / 2 \leqslant \phi \leqslant \pi / 2)$ of the $t-$ $\phi$ cylinder coordinate grid is shown flattened out and tilted back with respect to the vertical direction along $n$ (and the broken vertical line on the right edge of the plot) shown perpendicular to the horizontal $\phi$ coordinate axis (constant $t$ ), itself aligned with the unit vector $\bar{n}$ along the slicing spatial angular direction. The unit vector $\bar{m}$ is along the threading local rest space angular direction. Extending this direction counterclockwise around one loop of the cylinder leads to the change in the coordinate time equal to the synchronization gap for $m$. Doing the same for the spatial direction $\bar{u}_{\text {(car) }}$ for the Carter observer leads to the singleclock clock effect $\Delta t_{(\mathrm{geo})}$ for $m$. The worldlines of the various observers are labelled by their 4-velocities, and their successive crossing points with the pair of circular geodesics and with the pair of photon orbits characterize the clock and Sagnac effects, respectively, for the given observer. 
These formulae may be used to express the angular momentum (per unit mass)

$$
p_{\phi}=U_{\phi}=\Gamma \nu(U, n)_{\phi}=\Gamma g_{\phi \phi}\left(\zeta-\zeta_{(\mathrm{sl})}\right)
$$

of $U$ defined by the rotational Killing vector $\partial_{\phi}$ and its Killing energy (per unit mass) $\mathcal{E}=-U_{t}=M^{-1} \gamma(U, m)$ defined by the Killing vector $\partial_{t}$, both conserved for geodesic motion. These are related to the coordinate gamma factor by the identity $-1=U_{\alpha} U^{\alpha}=$ $\Gamma\left(-\mathcal{E}+\zeta p_{\phi}\right)$ in the timelike case and $0=P_{\alpha} P^{\alpha}=\Gamma_{\text {(null) }}\left(-\mathcal{E}+\zeta p_{\phi}\right)$ in the null case, where $\left(\mathcal{E}, p_{\phi}\right)=\left(-P_{t}, P_{\phi}\right)$. In the timelike case the ratio

$$
\bar{\zeta}=\frac{\mathcal{E}}{p_{\phi}}=-\frac{g_{t t}+\zeta g_{\phi t}}{g_{t \phi}+\zeta g_{\phi \phi}}
$$

defines the angular velocity of the circular orbit along $\bar{U}$ orthogonal to $U$. For the null case, one has the limiting case $\bar{\zeta}_{ \pm}=\zeta_{ \pm}$given by this same formula.

Note from equation (3.7) that the coordinate angular velocity of the slicing observers is just the average of the two limiting angular velocities

$$
\zeta_{(\mathrm{sl})}=\zeta_{(\mathrm{nmp})}=\left(\zeta_{-}+\zeta_{+}\right) / 2=-N^{\phi},
$$

which is just equation (33.16) of Misner et al [14] in the specific context of the Kerr spacetime. Their exercise (33.3) following the discussion of Bardeen [16] applies to the general (orthogonally transitive) stationary axially symmetric case [17], so that one may interpret the slicing observers as the locally non-rotating observers with respect to the Sagnac effect [18-23]. They experience no Sagnac effect for the oppositely directed accelerated photons constrained by mirrors or fibre optical cable to remain on a given circular orbit, meaning that the alternating meeting points of these photons ('null meeting points', occurring every other crossing of their worldlines) lie on the same observer worldline. They are also called the 'zero angular momentum observers' (ZAMOs), since they are orthogonal to the angular Killing vector and therefore have vanishing angular momentum.

Similarly, the spatial $\phi$ direction with respect to the original threading observers is defined by the angular velocity $\bar{\zeta}_{\text {(th) }}=M_{\phi}^{-1}$. By direct calculation from (3.7) one finds the following result:

$$
\bar{\zeta}_{\text {(th) }}{ }^{-1}=\left(\zeta_{-}{ }^{-1}+\zeta_{+}{ }^{-1}\right) / 2=M_{\phi} .
$$

This establishes a complementary relation between the null angular velocity reciprocals and the threading shift 1 -form coordinate component in direct analogy to the slicing relation between the angular velocities themselves and the shift vector coordinate component (3.14).

Both of these relationships are associated with the null path synchronization of nearby observers illustrated in figure 2 , where nearby slicing observer worldlines are identified with paths in the tangent space. In the left-hand diagram, the slicing observers are defined so that constant time coordinate lines are synchronized. The null paths (photon worldlines) starting at the origin from the middle observer at $t=0$ are reflected from either of the nearby observers at time $t=\Delta t$ and return to the middle observer at time $t=2 \Delta t$. This synchronizes the reflection events with the location of the middle observer at time $t=\Delta t$, at the event identified with the tip of the average vector $X_{0}$ in the tangent space, and leads to the angular velocity of the middle slicing observer being equal to the average of the photon angular velocities, yielding the formula (3.14) for the sign-reversed angular shift vector component. In the righthand diagram, the threading observers instead follow lines of constant $\phi$ in the tangent space, and synchronize themselves along the orthogonal direction. The middle observer at $\phi=\Delta \phi$ synchronizes the origin on the left observer at $\phi=0$ with the event $Y_{0}$ on his worldline 

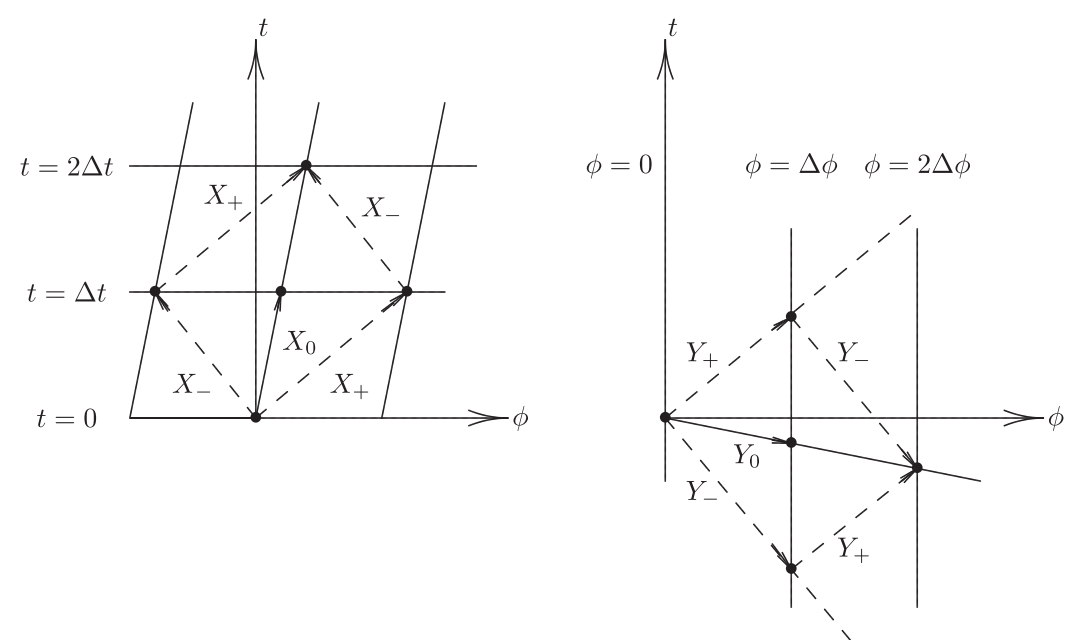

Figure 2. The null synchronization process associated with the interpretation of the shift fields in the slicing and threading points of view. Identifying nearby events with points in the tangent space, corotating $(+)$ and counterrotating $(-)$ photon paths are marked by broken lines. In the left-hand diagram, the slicing observers are locally synchronized by the $\phi$ coordinate lines, while in the right-hand diagram the threading observers are locally synchronized orthogonally to the $t$-coordinate lines, both within the $t-\phi$ cylinders, as explained in the text. The null vectors and their averages are given by $X_{ \pm}=\Delta t\left(\partial_{t}+\zeta_{ \pm} \partial_{\phi}\right), X_{0}=\left(X_{+}+X_{-}\right) / 2, Y_{ \pm}=\Delta \phi\left(\partial_{\phi}+\bar{\zeta}_{ \pm}^{-1} \partial_{t}\right)$, $Y_{0}=\left(Y_{+}+Y_{-}\right) / 2$.

corresponding to half the photon round trip to and from the left observer. This leads to the inverse angular velocity $\bar{\zeta}_{(\text {th })}{ }^{-1}$ of the spatial synchronization direction being the average of the corresponding inverse angular velocities $\bar{\zeta}_{ \pm}^{-1}=\zeta_{ \pm}^{-1}$ of the photons, yielding the angular shift 1-form component formula (3.15).

The threading discussion also applies to the new threading of the generic observer. This diagram shows how the threading shift characterizes the desynchronization effect, which is nothing more than the local tangent space version of the Sagnac effect through the local photon synchronization process. The coordinate time difference $\Delta t$ between the $\phi$-coordinate line and the right observer at the event $2 Y_{0}$, extrapolated along the two oppositely directed photon circular orbits, becomes the Sagnac effect, just twice the extrapolation of the synchronization gap between the $\phi$ coordinate line and the event $Y_{0}$. This result, which in turn is closely connected with the various clock effects, will be shown explicitly below.

\section{Other special observer families}

In the same way that the slicing observers are characterized as the 'null meeting point' observers, one can introduce the 'geodesic meeting point' observers $[7,8,24]$ that see no time delay between the arrival of oppositely rotating circular geodesics, i.e. the same period for the two orbits as seen by that observer. Their angular velocity, like in the null case, is just the average of the oppositely signed angular velocities $\dot{\phi}_{-}<0<\dot{\phi}_{+}$of these two circular orbits

$$
\zeta_{\text {(gmp) }}=\left(\dot{\phi}_{+}+\dot{\phi}_{-}\right) / 2=\left(\left|\dot{\phi}_{+}\right|-\left|\dot{\phi}_{-}\right|\right) / 2
$$

as a short calculation shows $\left(\dot{\phi}_{+} t=2 \pi+\Delta \phi,-\dot{\phi}_{-} t=2 \pi-\Delta \phi\right.$, subtract and solve for $\Delta \phi$ to find coefficient of $t$ as the desired angular velocity). This meeting point condition is valid for 
any pair of oppositely signed angular velocities $\left(\zeta_{1}, \zeta_{2}\right), \zeta_{1}<0<\zeta_{2}$, so that one can introduce

$$
\zeta_{(\mathrm{mp})}=\left(\zeta_{1}+\zeta_{2}\right) / 2=\left(\zeta_{2}-\left|\zeta_{1}\right|\right) / 2
$$

If $U_{1}, U_{2}, U_{(\mathrm{mp})}$ are the corresponding 4-velocities, then the meeting point observers see $U_{1}$ and $U_{2}$ moving with angular velocities of equal magnitude but opposite sign

$$
\zeta_{1}-\zeta_{(\mathrm{mp})}=-\left[\zeta_{2}-\zeta_{(\mathrm{mp})}\right]
$$

Because of the linear relation (3.9) between $\zeta$ and $v(U, n)$, the corresponding slicing relative velocities are also in the same averaging relation

$$
v\left(U_{(\mathrm{mp})}, n\right)=\left(v\left(U_{1}, n\right)+v\left(U_{2}, n\right)\right) / 2 .
$$

On the other hand, differences of angular velocities are proportional to differences of slicing relative velocities for the same reason

$$
v\left(U_{1}, n\right)-v\left(U_{2}, n\right)=N^{-1} g_{\phi \phi}^{1 / 2}\left(\zeta_{1}-\zeta_{2}\right) .
$$

Another family of preferred observers associated with the clock effects is the one for which the magnitude of the acceleration is extremal with respect to the angular velocity parameter of the circular orbit family [3,25-27]. In the equatorial plane in the Kerr spacetime, one has the inequalities

$$
\zeta_{(\mathrm{ext})}<\zeta_{(\mathrm{gmp})}<0<\zeta_{(\mathrm{nmp})}
$$

The first inequality is discussed in equations (4.24) and (4.25) of [3], while an explicit formula for the extremely accelerated observer angular velocity has been given by Semerák [6]

$$
\zeta_{(\mathrm{ext})}=\frac{r^{2}(r-3 \mathcal{M})-2 \mathcal{M} a^{2}-\sqrt{r^{4}(r-3 \mathcal{M})^{2}-4 \mathcal{M} a^{2} r^{3}}}{-2 \mathcal{M} a\left(3 r^{2}+a^{2}\right)},
$$

first stated for the variable $y=\zeta /(1-a \zeta)$ by de Felice [25,28]. Table 2 leads to the explicit simple formula

$$
\zeta_{(\mathrm{gmp})}=-a \mathcal{M} / r^{3} /\left(1-a^{2} \mathcal{M} / r^{3}\right)<0
$$

for the second angular velocity, while the last inequality follows from equation (3.14) and table 1. Note that both of these angular velocities have the same leading behaviour at large $r$, namely $-a \mathcal{M} / r^{3}$. Figure 1 shows the corresponding worldlines on the $t-\phi$ cylinder.

Table 2. Causal restrictions and geodesic and null conditions for circular orbits in the Kerr spacetime on the equatorial plane.

\begin{tabular}{ll}
\hline Threading region of validity & $r>r_{(\mathrm{erg})}=2 \mathcal{M}$ \\
Slicing region of validity & $r>r_{(\mathrm{h})}=\mathcal{M}+\sqrt{\mathcal{M}^{2}-a^{2}}$ \\
Timelike geodesics & $\dot{\phi}_{ \pm}=\frac{ \pm \sqrt{\mathcal{M} / r^{3}}}{1 \pm a \sqrt{\mathcal{M} / r^{3}}}$ \\
& $v\left(U_{ \pm}, m\right)^{\hat{\phi}}=\frac{\sqrt{\Delta}}{a \pm(r-2 \mathcal{M}) \sqrt{r / \mathcal{M}}}$ \\
& $v\left(U_{ \pm}, n\right)^{\hat{\phi}}=\frac{a^{2} \mp 2 a \sqrt{\mathcal{M} r}+r^{2}}{\sqrt{\Delta}(a \pm r \sqrt{r / \mathcal{M}})}$ \\
& $\zeta_{ \pm}=\frac{2 a \mathcal{M} \pm r \sqrt{\Delta}}{r^{3}+a^{2} r+2 a^{2} \mathcal{M}}$ \\
\hline
\end{tabular}




\section{Preliminary considerations}

The Sagnac effect [18-23] and the observer-dependent single-clock clock effect (its timelike analogue), both of which in turn are connected to the desynchronization effect, refer to the asymmetry in the arrival times of a pair of oppositely rotating null circular orbits or timelike geodesic circular orbits at a given radius as seen by a given rotating observer. These are illustrated in figure 1 (the Kerr equatorial plane) for the slicing (null meeting point) observers and threading observers (of the original coordinate grid), the geodesic meeting point observers, the geodesic observers, the extremely accelerated observers and the Carter observers. The forward and backward null orbits are labelled by $P_{ \pm}$, while the forward and backward geodesic orbits are labelled by $U_{ \pm}$, all starting at the same spacetime event at the origin of coordinates.

If $\left(\zeta_{1}, \zeta_{2}\right), \zeta_{1}<0<\zeta_{2}$, is the ordered pair of coordinate angular velocities of such a pair (either $\left(\zeta_{-}, \zeta_{+}\right)$for the Sagnac effect or $\left(\dot{\phi}_{-}, \dot{\phi}_{+}\right)$for the single-clock clock effect), and $\zeta$ is the angular velocity of a rotating observer with 4-velocity $U$ distinct from this pair, and if the observer has an intermediate angular velocity $\left(\zeta_{1}<\zeta<\zeta_{2}\right.$, only a restriction in the second case), one finds that the difference of the coordinate arrival times after one complete revolution with respect to this observer is

$$
\begin{aligned}
\Delta t & =\mathcal{S}\left(\zeta ; \zeta_{1}, \zeta_{2}\right)=t_{2}-t_{1}=2 \pi\left[1 /\left(\zeta_{2}-\zeta\right)-1 /\left(\zeta-\zeta_{1}\right)\right] \\
& =-4 \pi\left[\zeta-\left(\zeta_{1}+\zeta_{2}\right) / 2\right] /\left[\left(\zeta-\zeta_{1}\right)\left(\zeta-\zeta_{2}\right)\right], \\
& =-2 \pi \partial_{\zeta} \ln \left[\left(\zeta-\zeta_{1}\right)\left(\zeta-\zeta_{2}\right)\right] .
\end{aligned}
$$

If desired the coordinate time difference $\Delta t$ between the two hypersurfaces $t=t_{1}$ and $t=t_{2}$ at which the two crossings occur with the observer worldline can be converted into a proper time difference $\Delta \tau(U)$ measured by that observer using equation (3.6).

To understand the origin of these formulae, let both orbits and observer start at $t=0$ at the same point in spacetime. When the corotating orbit first meets the observer at time $t_{2}$ (equal to the observer-dependent coordinate period of that orbit), the observer has gone a coordinate angle distance $\zeta t_{2}$ while the corotating orbit has gone a coordinate angle distance $\zeta_{2} t_{2}$, which is one revolution plus the additional amount gone by the observer compared with the coordinate time line through the starting point, i.e. $\zeta_{2} t_{2}=2 \pi+\zeta t_{2}$ so $t_{2}=2 \pi /\left(\zeta_{2}-\zeta\right)$. The same consideration for the counterrotating orbit applies except that one must subtract $2 \pi$ from the observer angular distance, obtaining finally $t_{1}=2 \pi /\left(\zeta-\zeta_{1}\right)$. Their difference is the desired result.

Note that in the limits $\zeta \rightarrow \zeta_{1}$ and $\zeta \rightarrow \zeta_{2}$, the time difference $\mathcal{S}\left(\zeta ; \zeta_{1}, \zeta_{2}\right)$ goes to infinity since as the observer angular velocity approaches one of the two orbit values, it takes an increasingly longer time for that faster moving orbit to overtake the slightly slower observer by an extra revolution.

In terms of relative velocities $v(U, n) \equiv v, v\left(U_{1,2}, n\right) \equiv v_{1,2}$ and $v_{(\mathrm{mp})}=\left(v_{1}+v_{2}\right) / 2$ with respect to ZAMOs, the expressions for the general time interval for both the timelike and null cases, after converting velocity differences with (4.5), become

$\mathcal{S}\left(\zeta ; \zeta_{1}, \zeta_{2}\right)=-4 \pi \frac{g_{\phi \phi}^{1 / 2}}{N} \frac{v-v_{(\mathrm{mp})}}{\left(v-v_{1}\right)\left(v-v_{2}\right)}, \quad \mathcal{S}\left(\zeta ; \zeta_{+}, \zeta_{-}\right)=4 \pi \frac{g_{\phi \phi}^{1 / 2}}{N} \gamma^{2} \nu$.

Using these results, the observer that sees the same time interval both for a pair of oppositely rotating timelike particles and the corresponding photon pair can be easily found from the condition

$$
\mathcal{S}\left(\zeta ; \zeta_{1}, \zeta_{2}\right)=\mathcal{S}\left(\zeta ; \zeta_{+}, \zeta_{-}\right)
$$


This reduces to a quadratic equation in the slicing relative velocity of the observer

$$
v^{2}-2 v \frac{1+v_{1} v_{2}}{v_{1}+v_{2}}+1=0
$$

which always has two real solutions, one subluminal and the other superluminal [3]. One can rewrite this equation in the form

$$
\frac{v_{1}-v}{1-v v_{1}}=-\frac{v_{2}-v}{1-v v_{2}}
$$

which is the familiar relativistic addition of velocity formula applied to the two relative velocity differences, equivalent to

$$
v\left(U_{1}, U\right)=-v\left(U_{2}, U\right)
$$

Its simple content is that the observer ( $U$, corresponding to the subluminal solution $v$ ) that sees the same delay in the arrival times both of the particle and photon pairs also sees the corotating $\left(U_{2}\right)$ and counterrotating $\left(U_{1}\right)$ particles moving with equal magnitude but oppositely signed relative velocities. However, this just means that the observer 4-velocity is the normalized average of the two particle 4-velocities

$$
U=\frac{U_{1}+U_{2}}{\left\|U_{1}+U_{2}\right\|} \leftrightarrow \zeta=\left(\Gamma_{1} \zeta_{1}+\Gamma_{2} \zeta_{2}\right) /\left(\Gamma_{1}+\Gamma_{2}\right)
$$

as discussed after equation (4.8) of [3].

Turning this around, from the point of view of any fixed observer $(U)$, one therefore has a universal time delay for every pair of oppositely rotating timelike circular orbits which are seen symmetrically by that observer (relative velocities of equal magnitude but opposite sign), including the limiting case of the photon pair, independent of the common magnitude of the relative velocities of the pair.

\section{Single-clock clock effect}

For a pair of oppositely rotating timelike geodesics, one has

$$
\begin{aligned}
\Delta t_{\text {(geo) }}(U) & =\mathcal{S}\left(\zeta ; \dot{\phi}_{-}, \dot{\phi}_{+}\right)=-4 \pi\left[\zeta-\zeta_{(\mathrm{gmp})}\right] /\left[\left(\zeta-\dot{\phi}_{-}\right)\left(\zeta-\dot{\phi}_{+}\right)\right] \\
& =-2 \pi \partial_{\zeta} \ln \left[\left(\zeta-\dot{\phi}_{-}\right)\left(\zeta-\dot{\phi}_{+}\right)\right],
\end{aligned}
$$

while for the pair of oppositely rotating null orbits one has

$$
\begin{aligned}
\Delta t_{(\mathrm{null})}(U) & =\mathcal{S}\left(\zeta ; \zeta_{-}, \zeta_{+}\right)=-4 \pi\left[\zeta-\zeta_{(\mathrm{nmp})}\right] /\left[\left(\zeta-\zeta_{-}\right)\left(\zeta-\zeta_{+}\right)\right] \\
& =-2 \pi \partial_{\zeta} \ln \left[\left(\zeta-\zeta_{-}\right)\left(\zeta-\zeta_{+}\right)\right],
\end{aligned}
$$

recalling that

$$
\zeta_{(\mathrm{gmp})}=\left(\dot{\phi}_{+}+\dot{\phi}_{-}\right) / 2, \quad \zeta_{(\mathrm{nmp})}=\left(\zeta_{+}+\zeta_{-}\right) / 2=\zeta_{(\mathrm{sl})} .
$$

Each of these may be evaluated for any observer $U$. Figure 1 illustrates these two time delays for each of the various geometrically defined observers on the equatorial plane of the Kerr spacetime. Note that the null arrival time difference is proportional to the angular momentum (3.12) of $U$.

The difference between the arrival times of circularly rotating timelike geodesics and photons, as measured by a generic observer $U$, is simply related to the $\zeta$-derivative of the magnitude of the observer 4-acceleration $A(U)$

$$
\Delta t_{\text {(geo) }}(U)-\Delta t_{\text {(null) }}(U)=-2 \pi \partial_{\zeta} \ln \|A(U)\|,
$$


since by equation (4.3) of Bini et al [3] one has in the equatorial plane

$$
\|A(U)\|=\left|-\kappa(\phi, n)^{\hat{r}}\right| \frac{\left(\zeta-\dot{\phi}_{-}\right)\left(\zeta-\dot{\phi}_{+}\right)}{\left(\zeta-\zeta_{-}\right)\left(\zeta-\zeta_{+}\right)}
$$

where $\kappa(\phi, n)$ is a curvature factor independent of $\zeta$.

This relation gives the extremely accelerated observers $\left(\partial_{\zeta} \ln \|A(U)\|=0\right)$ the special property

$$
\Delta t_{(\mathrm{geo})}\left(U_{(\mathrm{ext})}\right)=\Delta t_{(\mathrm{null})}\left(U_{(\mathrm{ext})}\right),
$$

which must be added to their many other special properties arising in the discussion of inertial forces and gyroscope precession measurements [25,26]. In particular, the discussion after equation (5.3) shows that the extremal force observers must therefore see the oppositely rotating pair of geodesics moving with the same speed. However, the single-clock clock effect for those observers is negative for Kerr so the counterrotating geodesic arrives after the corotating one, as shown in figure 1.

The Sagnac time difference and the single-clock clock effect associated with the time intervals as seen by the threading observer $U=m(\zeta=0)$ are

$$
\begin{aligned}
& \Delta t_{\text {(null) }}(m)=\mathcal{S}\left(0 ; \zeta_{-}, \zeta_{+}\right)=4 \pi\left(\zeta_{-}{ }^{-1}+\zeta_{+}{ }^{-1}\right) / 2=4 \pi M_{\phi}, \\
& \Delta t_{\text {(geo) }}(m)=\mathcal{S}\left(0 ; \dot{\phi}_{-}, \dot{\phi}_{+}\right)=4 \pi\left(\dot{\phi}_{-}{ }^{-1}+\dot{\phi}_{+}{ }^{-1}\right) / 2=4 \pi a \quad[\text { Kerr }],
\end{aligned}
$$

where the final equality only holds for the Kerr spacetime.

The Sagnac effect $\Delta t_{\text {(null) }}(m)$ is positive (negative) when the threading observers corotate (counterrotate) with respect to the slicing observers $\left(\operatorname{sgn} \Delta t_{(\text {null })}=\operatorname{sgn} N^{\phi}=\operatorname{sgn} M_{\phi}\right)$. This is negative for Kerr (see table 1), where the slicing observers rotate forward around the hole compared with the threading observers which resist the so-called dragging of inertial frames, in contrast with the single-clock clock effect $\Delta t_{(\mathrm{geo})}(m)$ which is instead positive for Kerr.

The synchronization gap is the time difference which occurs during one spatial loop $C$ along the $\phi$-direction with respect to the threading observers. Integrating the differential condition of equation (2.7) along a counterclockwise trip (increasing $\phi$ ) one finds

$\Delta t_{(\mathrm{SG})}(m)=\int_{C} \mathrm{~d} t=\int_{0}^{2 \pi} M_{\phi} \mathrm{d} \phi=2 \pi M_{\phi}=2 \pi / \bar{\zeta}_{(\mathrm{th})}=\Delta t_{(\mathrm{null})}(m) / 2$.

The synchronization gap is exactly half the Sagnac time difference (see figure 1), with the factor of two coming from the fact that the Sagnac loop consists of two revolutions compared with the single revolution of the synchronization gap 'loop'. To understand this close connection, one must also approach the Sagnac time difference by integrating a differential condition, as will be done below. Note that this relation is true for a generic observer taken as the new threading observer, and so holds for the corresponding tilde quantities

$$
\Delta \tilde{t}_{(\mathrm{SG})}(U)=\int_{\tilde{C}} \mathrm{~d} \tilde{t}=\int_{0}^{2 \pi} \tilde{M}_{\tilde{\phi}} \mathrm{d} \tilde{\phi}=2 \pi \tilde{M}_{\tilde{\phi}}=\Delta \tilde{t}_{(\mathrm{null})}(U) / 2 .
$$

The analogous coordinate time difference for the geodesic case in the Kerr spacetime is the single-clock clock effect for the threading observers

$$
2 \pi / \bar{\zeta}_{(\mathrm{car})}=\Delta t_{(\mathrm{geo})}(m) / 2=2 \pi a
$$

and corresponds exactly to one threading loop of a circular curve which is spatial instead with respect to a Carter observer connecting the average-time-of-return point on the threading observer worldline with either return point on the same worldline. Thus the Carter observers 
in the single-clock clock effect correspond to the threading observers in the Sagnac effect in a certain sense. Figure 1 shows this loop, described by $t^{\prime}=t-a \phi=0$, where $t^{\prime}$ is a local helical time coordinate orthogonal to these observers in the equatorial plane only [17]. With $\phi^{\prime}=\phi$, one has a new local slicing for which $\mathrm{d} \phi^{\prime} / \mathrm{d} t^{\prime}=\zeta /(1-a \zeta)=y$ is the angular velocity variable of de Felice [25, 28].

One can express the threading observer synchronization gap, Sagnac effect, and singleclock clock effect with respect to the threading observers using a single formula in terms of either the angular velocity or threading relative velocity. The 1 -form

$$
\begin{aligned}
& {[v(U, m) \gamma(U, m)]^{-1} \bar{U}_{\alpha} \mathrm{d} x^{\alpha}=-M\left(\mathrm{~d} t-\zeta^{-1} \mathrm{~d} \phi\right)} \\
& \quad=-M\left(\mathrm{~d} t-M_{\phi} \mathrm{d} \phi\right)+\gamma_{\phi \phi}{ }^{1 / 2} / \nu(U, m)^{\hat{\phi}} \mathrm{d} \phi
\end{aligned}
$$

orthogonal to the 4-velocity $U$ restricts to zero its worldline segment $C$, so the threading observer proper travel time (proper period as seen by the observer) is

$$
\begin{aligned}
\tau(m)=M t & (m)=M \int_{C} \mathrm{~d} t=[\operatorname{sgn} \zeta] M \int_{0}^{2 \pi} \zeta^{-1} \mathrm{~d} \phi=2 \pi M /|\zeta| \\
= & {\left[\operatorname{sgn} v(U, m)^{\hat{\phi}}\right] \int_{0}^{2 \pi}\left[M M_{\phi}+\gamma_{\phi \phi}^{1 / 2} / v(U, m)^{\hat{\phi}}\right] \mathrm{d} \phi } \\
= & 2 \pi\left[\operatorname{sgn} v(U, m)^{\hat{\phi}}\right]\left[M M_{\phi}+\gamma_{\phi \phi}{ }^{1 / 2} / \nu(U, m)^{\hat{\phi}}\right] .
\end{aligned}
$$

Thus the difference in arrival times of two oppositely rotating orbits is then

$$
\begin{aligned}
\Delta \tau(m) & =M\left[t_{2}(m)-t_{1}(m)\right] \\
& =2 \pi\left[2 M M_{\phi}+\gamma_{\phi \phi}{ }^{1 / 2}\left[1 / v\left(U_{1}, m\right)^{\hat{\phi}}+1 / v\left(U_{2}, m\right)^{\hat{\phi}}\right]\right],
\end{aligned}
$$

correcting equation (7.10) of [3] by a misplaced factor of two.

These same considerations apply to a generic stationary observer $(U)$ and any circular orbit $\left(U_{1}\right)$, once they are expressed in terms of the new threading potentials and fields

$\tau_{1}(U)=\tilde{M} \tilde{t}_{1}(U)=\tilde{M} \int_{\tilde{C}} \mathrm{~d} \tilde{t}=\left[\operatorname{sgn}\left(\zeta_{1}-\zeta\right)\right] \tilde{M} \int_{0}^{2 \pi} 1 /\left(\zeta_{1}-\zeta\right) \mathrm{d} \tilde{\phi}=2 \pi \tilde{M} /\left|\zeta_{1}-\zeta\right|$.

As in the original threading case, the difference in arrival times of two oppositely rotating orbits $\left(U_{1}\right.$ and $\left.U_{2}\right)$ can be expressed in terms of the observer relative velocities as

$$
\begin{aligned}
\Delta \tau(U) & =\tilde{M}\left[\tilde{t}_{2}(U)-\tilde{t}_{1}(U)\right] \\
& =2 \pi\left[2 \tilde{M} \tilde{M}_{\tilde{\phi}}+\tilde{\gamma}_{\tilde{\phi} \tilde{\phi}}^{1 / 2}\left[1 / v\left(U_{1}, U\right)^{\hat{\tilde{\phi}}}+1 / v\left(U_{2}, U\right)^{\hat{\tilde{\phi}}}\right]\right]
\end{aligned}
$$

For relative velocities of equal magnitude but opposite sign, this reduces to the first term alone, which is the proper time synchronization gap $2 \Delta \tau_{(\mathrm{SG})}(U)$. For photons this is always true for any observer, identifying the Sagnac effect with the synchronization gap (for two loops) for that observer. Thus for the constant angular velocity orbits, the one-loop effect is just the local effect in the tangent space illustrated in figure 2, multiplied by the total angle $2 \pi$ of the loop.

Similarly, the extremely accelerated observers see the oppositely rotating circular geodesics with equal magnitude but oppositely signed relative velocities, so this difference in the arrival times that they measure is also entirely due to their own synchronization gap. This is in contrast with the Carter observers whose synchronization gap over a complete revolution with respect to the threading observers equals the single-clock clock effect for those latter observers, and so is a hybrid quantity. 


\section{Two-clock clock effects}

The two-clock clock effects, towards which recent attention has been directed $[2,5,6,9,10]$, can now be calculated easily from the formula (6.14), corrected for the new proper times. The period of the orbit of $U_{1}$ in terms of the proper time of $U_{1}$ only differs by replacing $\tilde{M}$ by $\Gamma_{1}^{-1}$ in equation (6.14). The two proper periods of the oppositely rotating geodesics $\left(U_{ \pm}\right)$after one revolution with respect to $U$ are therefore

$$
\tau\left(U_{ \pm}, U\right)=\tilde{t}_{ \pm}(U) / \Gamma_{ \pm}=2 \pi \Gamma_{ \pm}^{-1} /\left|\dot{\phi}_{ \pm}-\zeta\right| .
$$

The difference in the two proper periods of the oppositely rotating geodesics $\left(U_{ \pm}\right)$then becomes

$$
\begin{aligned}
\Delta \tau\left(U_{+}, U_{-}, U\right) & =\tilde{t}_{+}(U) / \Gamma_{+}-\tilde{t}_{-}(U) / \Gamma_{-}=2 \pi\left[\Gamma_{+}^{-1} /\left(\dot{\phi}_{+}-\zeta\right)-\Gamma_{-}^{-1} /\left(\zeta-\dot{\phi}_{-}\right)\right] \\
& =-2 \pi g_{\phi \phi}^{1 / 2}\left(\gamma_{+}^{-1}+\gamma_{-}^{-1}\right) \frac{v(U, n)-v\left(U_{(\mathrm{ext})}, n\right)}{\left[v(U, n)-v\left(U_{+}, n\right)\right]\left[v(U, n)-v\left(U_{-}, n\right)\right]},
\end{aligned}
$$

where in the last equation the angular velocities have been expressed in terms of the slicing relative velocities using (3.10) and equation (5.7) for the angular velocity of the extremely accelerated observers has been used [3]. Thus although they have different arrival times, the two oppositely rotating geodesics measure the same proper time period after one revolution with respect to the extremely accelerated observers, as shown by Semerák for the Kerr and van Stockum spacetimes [6]. This is true for any stationary axisymmetric spacetime, including the Gödel spacetime, which also has extremely accelerated observers [3].

Finally, the observer-independent two-clock clock effect results from specializing these equations to the geodesic meeting point observer, recovering the results of Tartaglia $[9,10]$ for the Kerr and van Stockum spacetimes. The proper periods between three successive crossings corresponding to each geodesic making a complete revolution (with respect to such an observer) are

$\tau\left(U_{ \pm}, U_{(\mathrm{gmp})}\right)=\tilde{t}_{ \pm}\left(U_{(\mathrm{gmp})}\right) / \Gamma_{ \pm}=2 \pi \Gamma_{ \pm}^{-1} /\left|\dot{\phi}_{ \pm}-\zeta_{(\mathrm{gmp})}\right|=4 \pi \Gamma_{ \pm}^{-1} /\left|\dot{\phi}_{+}-\dot{\phi}_{-}\right|$

and their difference is

$$
\Delta \tau\left(U_{+}, U_{-}, U_{(\mathrm{gmp})}\right)=4 \pi\left[\Gamma_{+}^{-1}-\Gamma_{-}^{-1}\right] /\left|\dot{\phi}_{+}-\dot{\phi}_{-}\right| .
$$

The latter result must be halved to compare with the result of Tartaglia [9, 10] for Kerr, since he considers two successive crossings of the geodesics.

Note also that the first meeting point after the pair of geodesics leave $\phi=0$ will be at $\pi$ plus half the amount of angle through which the geodesic meeting point observers have moved in one full revolution

$$
\phi_{(\mathrm{gmp})}=\pi+\frac{1}{2} \zeta_{(\mathrm{gmp})} \tilde{t}_{ \pm}\left(U_{(\mathrm{gmp})}\right)=\pi\left[1+\frac{\dot{\phi}_{+}+\dot{\phi}_{-}}{\dot{\phi}_{+}-\dot{\phi}_{-}}\right] .
$$

If $\dot{\phi}_{+}<\left|\dot{\phi}_{-}\right|$, as occurs in the Kerr case, the geodesic meeting point observers counterrotate with respect to the threading observers, since the counterrotating geodesics are moving faster with respect to the threading observers. This goes against the naive 'dragging of inertial frames' intuition $[2,29,30]$. The total angular difference after $n$ full revolutions would then be

$$
\Delta \phi_{(\mathrm{gmp})}=n \zeta_{(\mathrm{gmp})} \tilde{t}_{ \pm}\left(U_{(\mathrm{gmp})}\right)=2 n \pi \frac{\dot{\phi}_{+}+\dot{\phi}_{-}}{\dot{\phi}_{+}-\dot{\phi}_{-}},
$$

which is another quantity one might consider measuring.

Figure 3 compares the three clock effects for a typical radius in the equatorial plane of Kerr spacetime. 


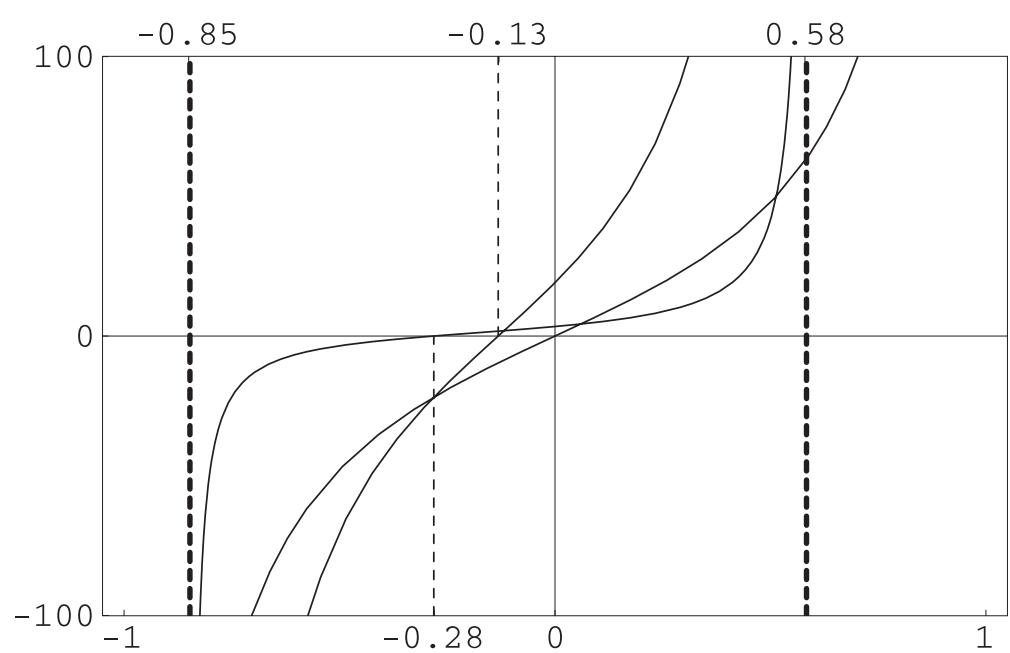

Figure 3. The behaviour of $\Delta \tau\left(U_{+}, U_{-}, U\right)$ (top right, middle curve), $\Delta t_{(\text {geo })}(U)$ (top right, left curve) and $\Delta t_{\text {(null) }}(U)$ (top right, right curve), at $r=4$ is shown as a function of the slicing relative velocity $v(U, n)$ in the case of circular orbits in the equatorial plane of the Kerr spacetime with $a=0.5, \mathcal{M}=1$. The two vertical asymptotes of $\Delta t_{(\mathrm{geo})}(U)$ correspond to the corotating and counterrotating geodesic velocities. Velocity values are indicated which correspond to zero values of $\Delta \tau\left(U_{+}, U_{-}, U\right)$ (extremely accelerated observers: $\left.v(U, n)=-0.28\right), \Delta t_{\text {(geo) }}(U)$ (geodesic meeting point observers: $v(U, n)=-0.13)$ and $\Delta t_{\text {(null) }}(U)$ (ZAMOs: $v(U, n)=0$ ). The crossing condition $\Delta t_{\text {(geo) }}(U)=\Delta t_{\text {(null) }}(U)$ also occurs at the extremely accelerated observer velocity.

\section{Explicit examples}

\subsection{Gödel}

For the Gödel spacetime the $t-\phi 2$-metric is

$$
\mathrm{d} s_{(t, \phi)}^{2}=-\mathrm{d} t^{2}+4 \Omega^{-1} s^{2} \mathrm{~d} t \mathrm{~d} \phi+2 \Omega^{-2} s^{2}\left(1-s^{2}\right) \mathrm{d} \phi^{2},
$$

where $s=\sinh (\Omega r / \sqrt{2})$ and $\Omega$ is the vorticity parameter of its fluid source [3]. The geodesic angular velocities and coordinate gamma factors are

$$
\begin{array}{ll}
\dot{\phi}_{+}=0, & \dot{\phi}_{-}=-2 \Omega /\left(1-2 s^{2}\right), \\
\Gamma_{+}=1, & \Gamma_{-}=\frac{1-2 s^{2}}{\sqrt{1-4 s^{2}\left(1+s^{2}\right)}},
\end{array}
$$

while the angular velocity of the extremely accelerated observer is

$$
\zeta_{(\mathrm{ext})}=-\frac{\Omega}{4 s^{4}}\left[1-2 s^{2}-\sqrt{1-4 s^{2}\left(1+s^{2}\right)}\right] .
$$

With these formulae, both the observer-dependent two-clock clock effect and the observerindependent two-clock clock effect then follow from equations (7.2) and (7.4).

\subsection{Kerr-Taub-NUT equatorial plane}

For the Kerr-Taub-NUT spacetime in the equatorial plane, the $t-\phi 2$-metric coefficients are [31]

$g_{t t}=-1+2 W, \quad g_{t \phi}=-2 a W, \quad g_{\phi \phi}=\left(r^{2}+a^{2}+\ell^{2}\right)+2 a^{2} W$, 
where $W=\left(\mathcal{M} r+\ell^{2}\right) /\left(r^{2}+\ell^{2}\right)$ and $\ell$ is the Taub-NUT parameter, which may be interpreted as the gravitomagnetic monopole moment of the source of the gravitational field or in other ways [32,33] and $J=\mathcal{M} a$ is the angular momentum, namely the gravitomagnetic dipole moment of the source.

The geodesic angular velocities are

$\dot{\phi}_{ \pm}^{-1}=a\left(1+\frac{2 \ell^{2} r}{\mathcal{M}\left(r^{2}-\ell^{2}\right)}\right) \pm \sqrt{\frac{r^{3}}{\mathcal{M}}}\left[\frac{\left(r^{2}+\ell^{2}\right)^{2}}{r^{2}\left(r^{2}-\ell^{2}\right)}+\frac{2 a^{2} \ell^{2}}{r^{2}\left(r^{2}-\ell^{2}\right)}\left(1+\frac{2 \ell^{2} r}{\mathcal{M}\left(r^{2}-\ell^{2}\right)}\right)\right]^{1 / 2}$,

which in the limit $r \rightarrow \infty$, together with their coordinate gamma factors, become

$$
\begin{aligned}
& \dot{\phi}_{ \pm}^{-1} \approx a \pm \sqrt{\frac{r^{3}}{\mathcal{M}}} \pm \frac{3}{2} \frac{\ell^{2}}{\sqrt{\mathcal{M} r}}+\frac{2 a \ell^{2}}{\mathcal{M} r} \\
& \Gamma_{ \pm} \approx 1+\frac{3}{2} \frac{\mathcal{M}}{r}+\frac{\ell^{2}}{r^{2}}+\frac{27}{8} \frac{\mathcal{M}^{2}}{r^{2}} \mp 3 \frac{a}{r}\left(\frac{\mathcal{M}}{r}\right)^{3 / 2},
\end{aligned}
$$

while the null meeting point, geodesic meeting point and extremely accelerated observer angular velocities are

$$
\begin{aligned}
& \zeta_{(\mathrm{nmp})} \approx \frac{a \mathcal{M}}{r^{3}}\left[2+\frac{2 \ell^{2}}{\mathcal{M} r}\right], \\
& \zeta_{(\mathrm{gmp})} \approx-\frac{a \mathcal{M}}{r^{3}}\left[1+\frac{2 \ell^{2}}{\mathcal{M} r}\right], \\
& \zeta_{(\mathrm{ext})} \approx-\frac{a \mathcal{M}}{r^{3}}\left[1+\frac{3 \mathcal{M}}{r}+\frac{2 \ell^{2}}{\mathcal{M} r}\right] .
\end{aligned}
$$

With these formulae, the various clock effects may be evaluated in this limit

$$
\begin{aligned}
& \Delta \tau_{(\mathrm{geo})}(m) \approx 4 \pi a\left[1+\frac{2 \ell^{2}}{\mathcal{M} r}\right], \\
& \Delta \tau_{(\mathrm{geo})}\left(U_{(\mathrm{ext})}\right) \approx-4 \pi a\left[0+\frac{3 \mathcal{M}}{r}+\frac{6 \mathcal{M}^{2}}{r^{2}}+\frac{4 \ell^{2}}{r^{2}}\right], \\
& \Delta \tau\left(U_{+}, U_{-}, m\right) \approx 4 \pi a\left[1+\frac{3 \mathcal{M}}{2 r}+\frac{2 \ell^{2}}{\mathcal{M} r}\right], \\
& \Delta \tau\left(U_{+}, U_{-}, U_{(\mathrm{gmp})}\right) \approx 4 \pi a\left[0+\frac{3 \mathcal{M}}{r}+\frac{9 \mathcal{M}^{2}}{2 r^{2}}+\frac{4 \ell^{2}}{r^{2}}\right] .
\end{aligned}
$$

Note that the difference between the single-clock and two-clock threading clock effects as well as the extremely accelerated observer single-clock effect and the clock-independent effect are suppressed by the factor $\mathcal{M} / r$, so experimentally there is really only one clock effect to be measured for solar system scenarios. Moreover, it turns out that the angular difference

$$
\Delta \phi_{(\mathrm{gmp})} \approx-\pi a \sqrt{\frac{\mathcal{M}}{r^{3}}}\left[1+\frac{2 \ell^{2}}{\mathcal{M} r}-\frac{3 \ell^{2}}{2 r^{2}}\right]
$$

is negligibly small in realistic experimental situations in the solar system.

The fact that both the gravitomagnetic monopole and dipole moments of the source of the gravitational field for this spacetime are non-zero makes it an interesting example for examining the clock effects. Note that only $\ell^{2}$ enters the metric in the equatorial plane, so the 
sign of $\ell$ is unimportant for these effects. For $r \gg \mathcal{M}$ and $r \gg \ell^{2} / \mathcal{M}$ the threading observer clock effects behave like

$$
4 \pi a=4 \pi \frac{J}{\mathcal{M} c^{2}},
$$

which is independent of the gravitational constant $G$ and the orbital radius $r[4,5,30,34]$ and has a value of about $10^{-7} \mathrm{~s}$ for the Earth. However, there are experimental difficulties that must be overcome before there is any hope of measuring this effect $[9,10,35,36]$.

Note that the gravitomagnetic clock effect may be extended to general orbits based on the idea of 'azimuthal closure' $[4,5,30,34]$. This also makes it clear that the definition of the azimuthal angle is crucial for the determination of this effect. In principle, one can use it to measure the rotation rate of the astronomical reference frame that is used for the interpretation of observations relative to the underlying spacetime with respect to which the source has intrinsic specific angular momentum $a$ [37].

\section{Conclusion}

The various gravitomagnetic clock effects, the Sagnac effect and the desynchronization effect for circularly rotating orbits in stationary axisymmetric spacetimes are all closely related. A relative observer analysis has shown how these effects can be studied together and how they are encoded into the symmetry-adapted coordinates. Special observer families then arise naturally. Extremely accelerated observers are shown to be the observer family for which the single-clock clock effect and Sagnac effects both agree since they both reduce to the synchronization gap, while their two-clock clock effect vanishes, as previously shown by Semerák. On the other hand, the geodesic meeting point observers are those for which the single-clock clock effect vanishes in direct analogy with the locally non-rotating observers (null meeting point observers) for which the Sagnac effect vanishes.

\section{Acknowledgments}

The authors are grateful to the organizers of the Spanish Relativity Meeting EREs2000 that led to this collaboration.

\section{References}

[1] Jantzen R T, Carini P and Bini D 1992 Ann. Phys., NY 2151

[2] Mashhoon B, Gronwald F and Lichtenegger H I M 2000 Testing Relativistic Gravity in Space ed C Lämmerzahl, C W F Everitt and F W Hehl (Berlin: Springer)

[3] Bini D, Carini P and Jantzen R T 1997 Int. J. Mod. Phys. D 6143

[4] Cohen J M and Mashhoon B 1993 Phys. Lett. A 181353

[5] Bonnor W B and Steadman B R 1999 Class. Quantum Grav. 161853

[6] Semerák O 1999 Class. Quantum Grav. 163769

[7] Mitskevich N V and Pulido Garcia I 1970 Sov. Phys. Dokl. 15591 (see [8])

[8] Ferrari V and Mashhoon B 1984 Phys. Rev. D 30295

[9] Tartaglia A 2000 Class. Quantum Grav. 17783

[10] Tartaglia A 2000 Class. Quantum Grav. 172381

[11] Carter B 1968 Commun. Math. Phys. 10280

[12] Kramer D, Stephani H, Herlt E and MacCallum M A H 1980 Exact Solutions of Einstein's Theory ed E Schmutzer (Cambridge: Cambridge University Press)

[13] Znajek R L 1977 Mon. Not. R. Astron. Soc. 175457

[14] Misner C W, Thorne K S and Wheeler J A 1973 Gravitation (New York: Freeman) 
[15] Bazański S L 1997 Mathematics of Gravitation Part II, Gravitational Wave Detection (Banach Center Publications vol 41) (Warsaw: Polish Academy of Sciences) p 273

[16] Bardeen J M 1970 Astrophys. J. 16271

[17] Greene R D, Schücking E L and Vishveshwara C V 1975 J. Math. Phys. 16153

[18] Post E J 1967 Rev. Mod. Phys. 39475

[19] Ashtekar A and Magnon A 1975 J. Math. Phys. 16341

[20] Henriksen R H and Nelson L A 1985 Can. J. Phys. 631393

[21] Anderson R, Bilger H R and Stedman G E 1994 Am. J. Phys. 62975

[22] Stedman G E 1985 Contemp. Phys. 26311

[23] Stedman G E 1997 Rep. Prog. Phys. 60615

[24] Mitskievich N V and Zaharow V N 1970 Doklady Akad. Nauk. SSSR. 195321 (in Russian)

Mitskievich N V 1996 Relativistic Physics in arbitrary reference frames Preprint gr-qc/9606051

[25] de Felice F 1994 Class. Quantum Grav. 111283 de Felice F 1995 Class. Quantum Grav. 121119

[26] Semerák O 1996 Gen. Rel. Grav. 281151

[27] Page D 1998 Class. Quantum Grav. 151669

[28] de Felice F and Usseglio-Tomasset S 1991 Class. Quantum Grav. 81871

[29] Rindler W 1997 Phys. Lett. A 23325

[30] Mashhoon B, Gronwald F and Theiss D S 1999 Ann. Phys., Lpz. 8135

[31] Miller J G 1973 J. Math. Phys. 14486

[32] Bonnor W B 1992 Gen. Rel. Grav. 24551

[33] Bradley M, Fodor G, Gergely L A, Marklund M and Perjés Z 1999 Class. Quantum Grav. 161667

[34] Mashhoon B and Santos N O 2000 Ann. Phys., Lpz. 949

[35] Lichtenegger H I M, Gronwald F and Mashhoon B 2000 Adv. Space Res. 251255

[36] Iorio L 2000 Int. J. Mod. Phys. D to appear (Iorio L 2000 Preprint gr-qc/0007014)

Iorio L 2000 Celest. Mech. submitted (Iorio L 2000 Preprint gr-qc/0007057)

[37] Wolf P 1999 Private communication to B Mashhoon 